\begin{tabular}{|c|c|}
\hline Title & Finite element beam propagation method with perfectly matched layer boundary conditions \\
\hline Author(s) & Koshiba, Masanori; Tsuji, Y asuhide; Hikari, Masafumi \\
\hline Citation & $\begin{array}{l}\text { IEEE TRA NSA CTIONS ON MA GNETICS, 35(3), 1482-1485 } \\
\text { https://doi.org/10.1109/20.767247 }\end{array}$ \\
\hline Issue Date & 1999-05 \\
\hline Doc URL & http:/hdl .handle.net/2115/5581 \\
\hline Rights & $\begin{array}{l}\text { O1999 IEEE. Personal use of this material is permitted. However, permission to reprint/republish this material for } \\
\text { advertising or promotional purposes or for creating new collective works for resale or redistribution to servers or lists, } \\
\text { or to reuse any copyrighted component of this work in other works must be obtained from the IEEE." }\end{array}$ \\
\hline Type & article \\
\hline File Information & IT M35-3.pdf \\
\hline
\end{tabular}

Instructions for use 


\title{
Finite Element Beam Propagation Method with Perfectly Matched Layer Boundary Conditions
}

\author{
Masanori Koshiba, Yasuhide Tsuji, and Masafumi Hikari \\ Division of Electronics and Information Engineering, Hokkaido University \\ Kita-13, Nishi-8, Kita-Ku, Sapporo 060-8628, Japan
}

\begin{abstract}
The perfectly matched layer boundary condition is incorporated into the beam propagation method based on a finite element scheme. To show the validity and usefulness of this approach, numerical results are shown for a double $U$-bend and linear tapers with different index contrast and opening angle.
\end{abstract}

Index terms - beam propagation method, finite element method, perfectly matched layer, wide-angle beam propagation, optical waveguide analysis

\section{INTRODUCTION}

The beam propagation method (BPM) is at the present the most widely used for the study of light propagation in longitudinally varying waveguides. Especially a recently developed finite element BPM (FE-BPM) using the Padé approximation [1], [2] can give very accurate results without increasing computational effort even if the wide-angle propagation is treated.

One of the key issues in implementing FE-BPM to study light propagation in finite spatial domain is the boundary condition at the computational window (CW) edges. Berenger [3] has recently introduced the concept of a perfectly matched layer (PML) for reflectionless absorption of electromagnetic waves which is successfully applied to the finite difference BPM (FDBPM) [4]. Unfortunately, since Berenger's PML technique involves a modification of Maxwell's equations based on the splitting of the fields components into two subcomponents, these non-Maxwellian equations do not have a desirable form for finite element (FE) formulations. More recently, Pekel and Mittra [5] have presented a new version of PML vyhich does not involve the field splitting and is suitable for $\mathrm{FE}$ formulations.

In this paper the revised PML is, for the first time, incorporated into FE-BPM. To show the validity and usefulness of this approach, numerical results are shown for a double U-bend and linear tapers with different index contrast and opening angle.

Manuscript received June 1, 1998

M. Koshiba, e-mail koshiba@ice.eng.hokudai.ac.jp, phone +81-11-7066541, fax +81-11-706-7892; Y. Tsuji, e-mail tsuji@ice.eng.hokudai.ac.jp, phone +81-11-706-6542; M. Hikari, e-mail hikari@ice.eng.hokudai.ac.jp, phone $+81-11-706-6545$.

\section{BASIC EQUATION}

We consider a planar (two-dimensional) optical waveguide, where $y$ and $z$ are the transverse and propagation directions, respectively, and there is no variation in the $x$ direction $(\partial / \partial x \equiv 0)$. With these assumptions and the transverselyscaled version of PML [5] we get the following basic equation:

$$
\frac{\partial}{\partial y}\left(\frac{p}{s} \frac{\partial \Phi}{\partial y}\right)+s \frac{\partial}{\partial z}\left(p \frac{\partial \Phi}{\partial z}\right)+k_{0}^{2} q s \Phi=0
$$

with

$$
\begin{array}{cll}
\Phi=E_{x}, & p=1, \quad q=n^{2} & \text { for TE modes } \\
\Phi=H_{x}, & p=1 / n^{2}, \quad q=1 \quad \text { for TM modes } \\
& s=1-j \frac{\sigma_{e}}{\omega \varepsilon_{0} n^{2}}=1-j \frac{\sigma_{m}}{\omega \mu_{0}}
\end{array}
$$

where $E_{x}$ and $H_{x}$ are the $x$ components of the electric and magnetic fields, respectively, $\omega$ is the angular frequency, $\varepsilon_{0}$ and $\mu_{0}$ are the permittivity and permeability of free space, respectively, $n$ is the refractive index, $k_{0}$ is the free-space wavenumber, and $\sigma_{e}$ and $\sigma_{m}$ are the electric and magnetic conductivities of PML, respectively.

The relation (4) is required to satisfy the PML impedance matching condition

$$
\frac{\sigma_{e}}{\varepsilon_{0} n^{2}}=\frac{\sigma_{m}}{\mu_{0}}
$$

which means that the wave impedance of a PML medium exactly equals that of the adjacent medium with refractive index $n$ in the $\mathrm{CW}, \sqrt{\mu_{0} /\left(\varepsilon_{0} n^{2}\right)}$, regardless of the angle of propagation or frequency. In the PML medium, a parabolic profile is usually assumed for the conductivities, and thus, the parameter $s$ is written as

$$
s= \begin{cases}1-j \frac{3 \lambda}{4 \pi n d}\left(\frac{\rho}{d}\right)^{2} \ln \frac{1}{R_{t}} & \text { in PML region } \\ 1 & \text { in non-PML region }\end{cases}
$$

where $\lambda$ is the free-space wavelength, $d$ is the PML thickness, $\rho$ is the distance from the beginning of PML, and $R_{t}$ is called the theoretical reflection coefficient [3]. 


\section{FINITE ELEMENT DISCRETIZATION}

Substituting a solution of the form

$$
\Phi(y, z)=\phi(y, z) \exp \left(-j k_{0} n_{0} z\right)
$$

into (1), we obtain the following equation for the slowly varying complex amplitude $\phi$ :

$p s \frac{\partial^{2} \phi}{\partial z^{2}}-2 j k_{0} n_{0} p s \frac{\partial \phi}{\partial z}+\frac{\partial}{\partial y}\left(\frac{p}{s} \frac{\partial \phi}{\partial y}\right)+k_{0}^{2} s\left(q-n_{0}^{2} p\right) \phi=0$

where $n_{0}$ is the reference refractive index, and the term $\partial p / \partial z$ is neglected for TM modes.

Dividing the waveguide cross-section into quadratic (second-order) line elements and applying the standard FE technique to (8), we obtain

$$
\begin{aligned}
& {[M] \frac{d^{2}\{\phi\}}{d z^{2}}-2 j k_{0} n_{0}[M] \frac{d\{\phi\}}{d z}} \\
& \quad+\left([K]-k_{0}^{2} n_{0}^{2}[M]\right)\{\phi\}=\{0\}
\end{aligned}
$$

where $\{\phi\}$ is the global electric or magnetic field vector, $\{0\}$ is a null vector, and the $\mathrm{FE}$ matrices $[K]$ and $[M]$ are given by

$$
\begin{gathered}
{[K]=\sum_{e} \int_{e}\left[k_{0}^{2} q s\{N\}\{N\}^{T}-\frac{p}{s} \frac{d\{N\}}{d y} \frac{d\{N\}^{T}}{d y}\right] d y} \\
{[M]=\sum_{e} \int_{e} p s\{N\}\{N\}^{T} d y}
\end{gathered}
$$

Here, $\{N\}$ is the shape function vector, $T$ denotes a transpose, and $\sum_{e}$ extends over all different elements.

Utilizing the Padé approximation [1],[2],[6], (9) is reduced to

$$
-2 j k_{0} n_{0}[\tilde{M}] \frac{d\{\phi\}}{d z}+\left(\left[I_{i}\right]-k_{0}^{2} n_{0}^{2}[M]\right)\{\phi\}=\{0\}
$$

with

$$
[\tilde{M}]=[M]+\frac{1}{4 k_{0}^{2} n_{0}^{2}}\left([K]-k_{0}^{2} n_{0}^{2}[M]\right)
$$

Applying the Crank-Nicholson algorithm for the propagation direction $z$ to (12) yields

$$
[A]_{i}\left\{\phi_{\Delta z}\right\}_{i}=[B]_{i}\{\phi\}_{i}
$$

with

$$
\begin{aligned}
& {[A]_{i}=-2 j k_{0} n_{0, i}[\tilde{M}]_{i}+0.5 \Delta z\left([K]_{i}-k_{0}^{2} n_{0, i}^{2}[M]_{i}\right)} \\
& {[B]_{i}=-2 j k_{0} n_{0, i}[\tilde{M}]_{i}-0.5 \Delta z\left([K]_{i}-k_{0}^{2} n_{0, i}^{2}[M]_{i}\right)}
\end{aligned}
$$

where $\Delta z$ is the propagation step size, $\{\phi\}_{i}$ and $\left\{\phi_{\Delta z}\right\}_{i}$ are the input and output field vectors at the $i$ th propagation step, respectively, and the reference refractive index $n_{0, i}$ and the FE mesh are adaptively renewed at each propagation step [2]. A temporary input field vector on the next $(i+1)$ th mesh, $\left\{\phi^{\prime}\right\}_{i+1}$, is easily obtained by quadratic interpolation using the

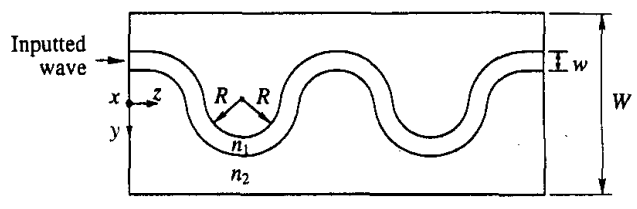

Fig. 1 U-bent optical waveguide.

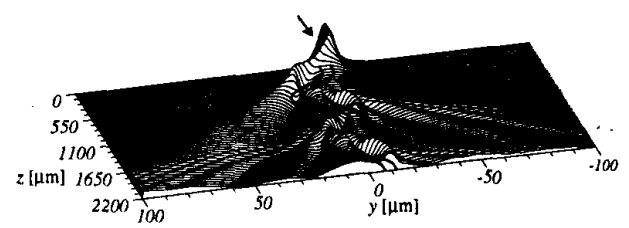

(a)

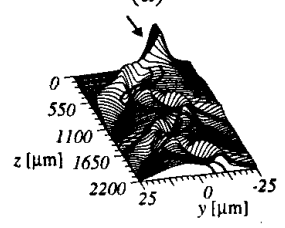

(b)

Fig. 2 Field evolution with computational window size of (a) $W=200 \mu \mathrm{m}$ and (b) $W=50 \mu \mathrm{m}$.

values of $\left\{\phi_{\Delta z}\right\}_{i}$. Noting that the transmitted power $P$ is given by

$$
P=\frac{1}{2} \int_{-\infty}^{\infty} p|\phi|^{2} d y
$$

an actual input field vector on the $(i+1)$ th mesh, $\{\phi\}_{i+1}$, is derived as

$$
\begin{aligned}
\{\phi\}_{i+1} & =\left\{\phi^{\prime}\right\}_{i+1} \frac{\int_{C W} p_{i}\left|\phi_{i}(y, z=i \Delta z)\right|^{2} d y}{\int_{C W} p_{i+1}\left|\phi_{i+1}^{\prime}(y, z=i \Delta z)\right|^{2} d y} \\
& =\left\{\phi^{\prime}\right\}_{i+1} \\
& \times \frac{\sum_{e}^{\prime}\left\{\phi_{\Delta z}\right\}_{i, e}^{\dagger}\left[\int_{e} p_{i}\{N\}\{N\}^{T} d y\right]\left\{\phi_{\Delta z}\right\}_{i, e}}{\sum_{e}^{\prime}\left\{\phi^{\prime}\right\}_{i+1, e}^{\dagger}\left[\int_{e} p_{i+1}\{N\}\{N\}^{T} d y\right]\left\{\phi^{\prime}\right\}_{i+1, e}}
\end{aligned}
$$

where $\left\{\phi_{\Delta z}\right\}_{i, e}$ and $\left\{\phi^{\prime}\right\}_{i+1, e}$ are the elemental field vectors, $\dagger$ denote complex conjugate and transpose, and $\sum_{e}^{\prime}$ extends over the elements within CW except PML regions.

\section{NUMERICAL RESULTS}

First, we consider a double U-bend as shown in Fig. 1, where the core index is $n_{1}=1.45$, the relative refractive index 


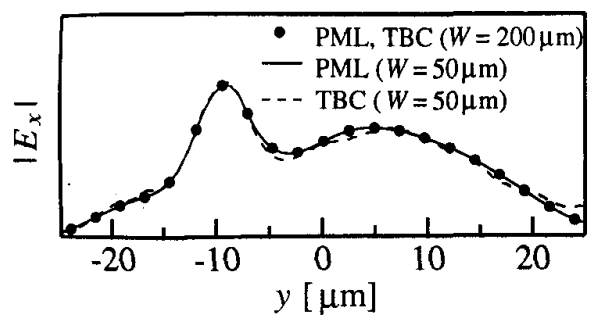

Fig. 3 Field pattern at $z=2200 \mu \mathrm{m}$.

difference is $\Delta=\left(n_{1}-n_{2}\right) / n_{1}=0.25 \%$, the core width is $w=5 \mu \mathrm{m}$, and the curvature radius is $R=3130 \mu \mathrm{m}$. Fig. 2 shows the electric field evolution of the fundamental TE wave propagating in the U-bend. A wavelength of $1.55 \mu \mathrm{m}$ is assumed, the PML thickness is $d=1 \mu \mathrm{m}$, the theoretical reflection coefficient is $R_{t}=10^{-5}$, and the propagation step size is $\Delta z=1 \mu \mathrm{m}$. We can see that the waves virtually disappear into the boundaries, irrespective of the $\mathrm{CW}$ size $W$. Fig. 3 shows the electric field $\left(E_{x}\right)$ pattern at the propagation distance of $z=2200 \mu \mathrm{m}$. When using the PML, the reflection from the $\mathrm{CW}$ edges is not observed. When using the transparent boundary condition (TBC) [7], on the other hand, we can see the field disturbance due to the spurious reflection.

Next, we consider a linear taper for the reciprocity test as shown in Fig. 4 [8] and compare numerical results with those of other propagation schemes in Tables I to III. Univ. Twente (FDBPM), Univ. Porto (FD-BPM), HHI (FD-BPM), ThomsonCFS (FD-BPM), AAR-UCL (FE-BPM), and Hokkaido Univ. (wide-angle FE-BPM presented here) use beam propagation methods, in other word, unidirectional algorithms, while Univ. Hagen (method of line BPM abbreviated as MoL-BPM), IREE Prague (mode expansion propagation method abbreviated as MEPM), and Univ. Gent (MEPM) use bidirectional algorithms taking account of reflected waves. In these tables $M$ is the number of discretization points within the $\mathrm{CW}$ (the number of modes for MEPM), $N$ is the number of propagation steps, a wavelength of $1.55 \mu \mathrm{m}$ is assumed, two opening angles of $0.1^{\circ}$ and $1.0^{\circ}$ are considered, and 'forward' or 'backward' propagation is defined in Fig. 4.

The fundamental TE and TM mode power losses in \% for the semiconductor cover case are listed in Tables I and II, respectively, where $n_{1}=3.3, n_{2}=3.17, n_{3}=3.17$, $w_{1}=0.2 \mu \mathrm{m}, w_{2}=0.1 \mu \mathrm{m}, W=20 \mu \mathrm{m}$, and $d=1.0 \mu \mathrm{m}$. Also in \%, the fundamental TE mode power losses for the air cover case are listed in Table III, where $n_{1}=3.3, n_{2}=3.17$, $n_{3}=1.0, w_{1}=0.8 \mu \mathrm{m}, w_{2}=0.4 \mu \mathrm{m}, W=20 \mu \mathrm{m}$, and $d=1.0 \mu \mathrm{m}$. In general, unidirectional algorithms are not able to fulfil the reciprocity criterion exactly [8]. However, our results (Hokkaido Univ.) obtained by the lowest discretization effort among the unidirectional algorithms fulfil fairly well the principle of reciprocity and agree approximately with the results of the bidirectional algorithms. The fundamental TEmode evolution as a function of propagation distance is plotted in Fig. 5. We can see that due to the reciprocity of propagation,

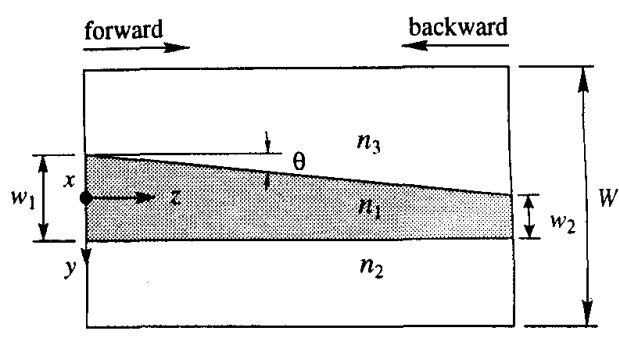

Fig. 4 Linearly tapered optical waveguide.

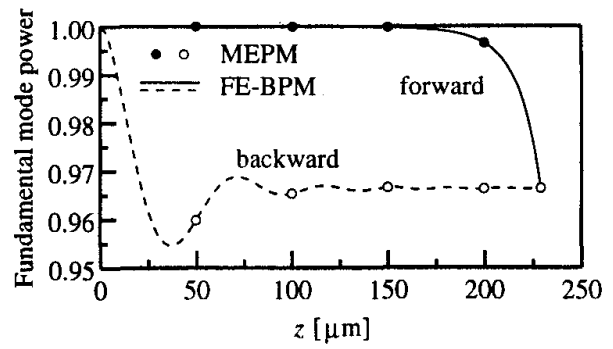

(a)

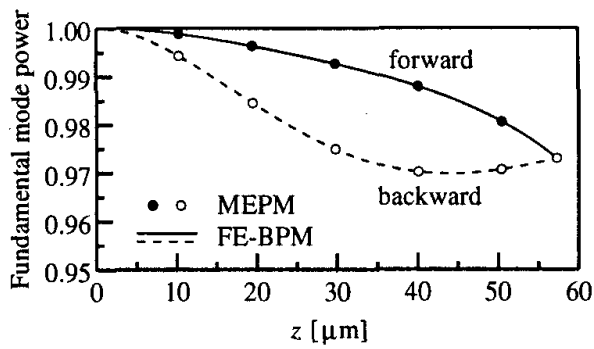

(b)

Fig. 5 The fundamental TE-mode power evolution for (a) the semiconductor cover case and (b) the air cover case $\left(\theta=0.1^{\circ}\right)$.

the end points of solid (forward) and dashed (backward) curves coincide, and that our results agree well with the results of MEPM from Univ. Gent [8].

\section{CONCLUSION}

The PML boundary condition was, for the first time, incorporated into the wide-angle FE-BPM based on the Padé approximation and its effectiveness was demonstrated. The FE-BPM with PML for three-dimensional optical waveguides is now under consideration.

\section{REFERENCES}

[1] M. Koshiba and Y. Tsuji, "A wide-angle finite-element beam propagation method," IEEE Photon. Technol. Lett, vol. 8, pp. 1208-1210, Sept. 1996. 
[2] Y. Tsuji, M. Koshiba, and T. Tanabe, "A wide-angle beam propagation method based on a finite element scheme," IEEE Trans. Magnet., vol. 33, pp. 1544-1547, Mar. 1997.

[3] J.-P. Berenger, "A perfectly matched layer for the absorption of electromagnetic waves," J. Comput. Phys., vol. 114, pp. 185-200, Oct. 1994.

[4] W.P. Huang, C.L. Xu, W. Lui, and K. Yokoyama, "The perfectly matched layer (PML) boundary condition for the beam propagation method," IEEE Photon. Technol. Lett., vol. 8, pp. 649-651, May 1996.

[5] Ü Pekel and R. Mittra, "An application of the perfectly matched layer (PML) concept to the finite element method frequency domain analysis of scattering problem," IEEE Microwave Guided Lett., vol. $\mathbf{5} p \mathrm{p}$. 258-260, Aug. 1995.
[6] G.R. Hadley, "Wide-angle beam propagation using Padé approximant operators," Opt. Lett., vol. 17, pp. 1426-1428, Oct. 1992.

[7] G.R. Hadley, "Transparent boundary condition for the beam propagation method," Opt. Lett., vol. 16, pp. 624-626, May 1991.

[8] J. Haes, R. Baets, C.M. Weinert, M. Gravert, H.P. Nolting, M.A Andrade, A. Leite, H.K. Bissessur, J.B. Davies, R.D. Ettinger, J. Ctyroky, E. Ducloux, F. Ratovelomanana, N. Vodjdani, S. Helfert, R. Pregla, F.H.G.M. Wijnands, H.J.W.M. Hoekstra, and G.J.M. Krijnen, "A comparison between different propagative schemes for the simulation of tapered step index slab waveguides," J. Lightwave Technol., vol. 14 pp. 1557-1569, June 1996

TABLE I CALCULATION CONDITIONS AND THE FUNDAMENTAL TE MODE POWER LOSS FOR THE SEMICONDUCTOR COVER CASE $\left(n_{1}=3.3, n_{2}=3.17, n_{3}=3.17, w_{1}=\right.$ $0.2 \mu \mathrm{m}$, and $\left.w_{2}=0.1 \mu \mathrm{m}\right)$

\begin{tabular}{|c|c|c|c|c|c|c|c|}
\hline \multirow{2}{*}{$\begin{array}{l}\text { Research } \\
\text { institute }\end{array}$} & \multirow{2}{*}{$M$} & \multicolumn{2}{|c|}{$N$} & \multicolumn{2}{|c|}{$\theta=0.1^{\circ}$} & \multicolumn{2}{|c|}{$\theta=1.0^{\circ}$} \\
\hline & & $\theta=0.1^{\circ}$ & $\theta=1.0^{\circ}$ & forward & backward & forward & backward \\
\hline Univ. Twente & 2048 & 100 & 10 & 2.68 & 2.70 & 8.82 & 8.81 \\
\hline Univ. Porto & 2500 & 100 & 10 & 2.69 & 2.70 & 8.81 & 8.81 . \\
\hline HHI & 1024 & 1061 & 106 & 2.50 & 2.50 & 8.90 & 8.80 \\
\hline Thomson-CFS & 1024 & 573 & 57 & 2.72 & 2.73 & 8.82 & 8.82 \\
\hline AAR-UCL & $120 / 170$ & 115 & 23 & 2.50 & 2.50 & 8.59 & 9.22 \\
\hline Hokkaido Univ. & 101 & 100 & 10 & 2.70 & 2.70 & 8.81 & 8.81 \\
\hline Univ. Hagen & 323 & 50 & 50 & 2.72 & 2.72 & 8.81 & 8.81 \\
\hline IREE Prague & 50 & 200 & 200 & 2.69 & 2.69 & 8.78 & 8.78 \\
\hline Univ. Gent & 51 & 20 & 20 & 2.69 & 2.69 & 8.82 & 8.82 \\
\hline
\end{tabular}

TABLE II CALCULATION CONDITIONS AND THE FUNDAMENTAL TM MODE POWER LOSS FOR THE SEMICONDUCTOR COVER CASE $\left(n_{1}=3.3, n_{2}=3.17, n_{3}=3.17, w_{1}=\right.$ $0.2 \mu \mathrm{m}$, and $\left.w_{2}=0.1 \mu \mathrm{m}\right)$

\begin{tabular}{|c|c|c|c|c|c|c|c|}
\hline \multirow{2}{*}{$\begin{array}{l}\text { Research } \\
\text { institute }\end{array}$} & \multirow{2}{*}{$M$} & \multicolumn{2}{|c|}{$N$} & \multicolumn{2}{|c|}{$\theta=0.1^{\circ}$} & \multicolumn{2}{|c|}{$\theta=1.0^{\circ}$} \\
\hline & & $\theta=0.1^{\circ}$ & $\theta=1.0^{\circ}$ & forward & backward & forward & backward \\
\hline Univ. Twente & 2048 & 100 & 10 & 3.37 & 3.40 & 9.15 & 9.14 \\
\hline Univ, Porto & 2500 & - & 10 & - & - & 9.70 & - \\
\hline HHI & - & - & - & 一 & - & - & - \\
\hline Thomson-CFS & 1024 & 573 & 57 & 3.39 & 3.40 & 9.13 & 9.15 \\
\hline AAR-UCL & $120 / 170$ & 115 & 23 & 3.17 & 3.17 & 9.43 & 9.64 \\
\hline Hokkaido Univ. & 101 & 100 & 10 & 3.39 & 3.41 & 9.20 & 9.23 \\
\hline Univ. Hagen & 324 & 50 & 50 & 3.39 & 3.45 & 9.15 & 9.20 \\
\hline IREE Prague & 50 & 200 & 200 & 3.37 & 3.37 & 9.10 & 9.10 \\
\hline Univ. Gent & 51 & 20 & 20 & 3.37 & 3.37 & 9.13 & 9.13 \\
\hline
\end{tabular}

TABLE III CALCULATION CONDITIONS AND THE FUNDAMENTAL TE MODE POWER LOSS FOR THE AIR COVER CASE $\left(n_{1}=3.3, n_{2}=3.17, n_{3}=1.0, w_{1}=0.2 \mu \mathrm{m}\right.$, and $\left.w_{2}=0.4 \mu \mathrm{m}\right)$

\begin{tabular}{|c|c|c|c|c|c|c|c|}
\hline \multirow{2}{*}{$\begin{array}{l}\text { Research } \\
\text { institute }\end{array}$} & \multirow{2}{*}{$M$} & \multicolumn{2}{|c|}{$N$} & \multicolumn{2}{|c|}{$\theta=0.1^{\circ}$} & \multicolumn{2}{|c|}{$\theta=1.0^{\circ}$} \\
\hline & & $\theta=0.1^{\circ}$ & $\theta=1.0^{\circ}$ & forward & backward & forward & backward \\
\hline Univ. Twente & 2048 & 400 & 400 & 3.19 & 3.36 & $17 . \overline{7}$ & 17.7 \\
\hline Univ. Porto & 2500 & 400 & 40 & 3.30 & 3.40 & 17.8 & 17.6 \\
\hline HHI & 1024 & 4241 & 424 & 3.30 & 3.50 & 18.4 & 18.5 \\
\hline Thomson-CFS & 1024 & 2290 & 229 & 3.46 & 3.39 & 17.9 & 17.7 \\
\hline AAR-UCL & $380 / 390$ & 558 & 92 & 2.50 & 1.83 & 14.7 & 14.3 \\
\hline Hokkaido Univ. & 301 & 400 & 40 & 3.36 & 3.36 & 17.7 & 17.7 \\
\hline Univ. Hagen & 334 & 60 & 60 & 3.64 & 3.64 & 18.5 & $1 \overline{8.6}$ \\
\hline IREE Prague & 50 & 200 & 200 & 3.40 & 3.40 & 17.7 & 17.7 \\
\hline Univ. Gent & 60 & 100 & 50 & 3.48 & 3.48 & 17.6 & 17.6 \\
\hline
\end{tabular}

